Revue scientifique sur la conception et l'aménagement de l'espace

\title{
De la défiance à la réconciliation : itinéraires des constructions urbaines et rivulaires des villes du Sillon lorrain (Nancy, Metz, Thionville)
}

From Mistrust to Reconciliation: Approaches in Urban and Riverside Development in the Sillon Lorrain Urban Cluster (Nancy, Metz, Thionville)

Denis Mathis, Anne Hecker, Jean-Pierre Husson et Kamila Bensaadi

\section{OpenEdition}

\section{Journals}

Édition électronique

URL : http://journals.openedition.org/paysage/739

DOI : $10.4000 /$ paysage.739

ISSN : 1969-6124

Éditeur :

École nationale supérieure du paysage de Versailles-Marseille, Institut national des sciences appliquées Centre Val de Loire - École de la nature et du paysage, École nationale supérieure d'architecture et de paysage de Bordeaux, École nationale supérieure d'architecture et de paysage de Lille, Agrocampus Angers

\section{Référence électronique}

Denis Mathis, Anne Hecker, Jean-Pierre Husson et Kamila Bensaadi, « De la défiance à la réconciliation : itinéraires des constructions urbaines et rivulaires des villes du Sillon lorrain (Nancy, Metz, Thionville) », Projets de paysage [En ligne], 20 | 2019, mis en ligne le 21 juin 2019, consulté le 28 novembre 2019. URL : http://journals.openedition.org/paysage/739 ; DOI : 10.4000/paysage.739

Ce document a été généré automatiquement le 28 novembre 2019.

Projets de paysage 


\title{
De la défiance à la réconciliation : itinéraires des constructions urbaines et rivulaires des villes du Sillon lorrain (Nancy, Metz, Thionville)
}

\author{
From Mistrust to Reconciliation: Approaches in Urban and Riverside \\ Development in the Sillon Lorrain Urban Cluster (Nancy, Metz, Thionville)
}

Denis Mathis, Anne Hecker, Jean-Pierre Husson et Kamila Bensaadi

1 Nombreuses sont les villes dont le dessein s'est construit autour d'un cours d'eau, depuis un site de pont ou d'un gué, constituant le point de départ de leur croissance, «cristallisant l'habitat» (Leguay, 2002), dans une rencontre avec le fleuve. De cette union naît une interaction entre le processus de «fabrique de la ville » et le cours d'eau, mais aussi un aménagement de la vallée avec ses zones humides et marécageuses, ses bras morts... Le couple ville-fleuve par ses aménagements construit des paysages originaux dans un rapport constant au fleuve, que celui-ci soit producteur de richesses ou au contraire soit perçu comme un danger. Cette ambivalence dans la connivence de la ville au fleuve doit s'observer et s'analyser sur un temps long. Ce dernier permet ainsi de souligner les phases de consolidation du couple ville-fleuve mais aussi les transitions (Hecker, 2017a; Mathis et Mathis, 2017), c'est-à-dire les transformations des rapports au sein de ce couple destinées à assurer les continuums d'un récit urbain.

2 Cette notion de couple se réfère à l'intrication, à l'harmonisation entre deux forces égales, parallèles et dirigées en sens contraire. Cette définition sied à la relation entretenue entre Nancy, la Meurthe et ses affluents (ruisseaux cataclinaux de rive gauche et sous-affluents de rive droite) et explique l'hydromorphologie urbaine de l'agglomération. Nancy a bénéficié de la proximité du cours d'eau assurant, par le pont de Malzéville (1498), le passage de la Meurthe; elle s'en est pourtant délibérément 
tenue à l'écart, et ce jusqu'à une période très récente. Le paysage urbain s'est donc construit en défiance de l'eau, laissant à la périphérie un espace dévolu à la Meurthe et à ses rives. Le noyau originel médiéval, puis la ville des princes de la Renaissance (René II puis Charles III), et enfin la ville des Lumières ont tourné le dos à la rivière, laissant un vaste glacis composé de prairies humides inondables et formant, au temps des fortifications nancéiennes, un hydrosystème défensif ${ }^{1}$.

3 La fabrique de l'urbain est intimement liée à la perception de l'hydrosystème fluvial. Ce concept est établi sur les interactions entre la dynamique fluviale, les processus biologiques et les activités humaines aux multiples échelles de temps et d'espace (Amoros et Petts, 1993). La dimension temporelle permet d'explorer les interactions entre le fleuve et la ville dont les impacts sont plus ou moins réversibles. Ainsi, cette étude cherche à analyser comment cette relation a évolué des origines de la ville médiévale jusqu'à la reconquête actuelle des fronts d'eau. Elle essaie de tenir compte des interactions permanentes entre les deux éléments : la ville et le cours d'eau par le biais de SIG historiques. Questionner ce couple sur un temps long (Guillerme, 1997) permet d'appréhender les relations entre ces deux systèmes dont la production paysagère n'est que le reflet. L'histoire de Nancy et de son hydrosystème est faite de rétroactions, de ruptures, d'accélérations mais également de permanences - par exemple la défiance par rapport aux crues ou la crainte des sévères étiages. Le couple souligne une mise en scène de la rivière qui s'effectue en trois actes. Successivement, il s'agit des époques médiévale et moderne, puis de la grande parenthèse industrielle des années 1870 à 1985, enfin les temps actuels. La synergie ville-rivière fut tardive, largement dictée par la conjoncture qui opère deux changements magistraux, l'un entre $1845^{2}$ et 1890 et le second à partir de $1982-1985^{3}$. Au-delà de la trajectoire nancéienne, c'est le lien ville-fleuve à l'échelle du Sillon lorrain qu'il est possible d'explorer, notamment en comparant Nancy et les itinéraires des villes de Metz et de Thionville dont les défiances vis-à-vis de la Moselle sont parfois assez similaires.

\section{Pour une approche géohistorique}

4 La démarche géohistorique, "extension du champ de la géographie aux espaces du passé » (Valette et Carozza, 2010), est entrée dans une nouvelle dimension grâce au SIG historique, véritable " outil d'histoire spatiale» (Gregory et Knowles, 2011). Cet outil, qui ne remplace pas mais accompagne données d'archives et analyses de terrain, permet en effet de capitaliser des données du passé (Pons, 2008), afin d'en comprendre et d'en analyser les multiples facettes, puis de mettre en œuvre des outils de prévision et de prospective qui découlent de ces connaissances mises en réseau et spatialisées.

5 L'appréhension des évolutions spatiales et temporelles de champs d'étude aussi mouvants et en constante évolution que les paysages de l'eau et le tissu urbain nous confronte en effet à plusieurs difficultés. Récentes ou historiques, les sources d'informations sont de natures diverses, et doivent être multipliées et croisées pour fournir l'information la plus exhaustive et précise possible. Le corpus documentaire constitué regroupe fréquemment des sources d'archives, des cartes et plans anciens, des photographies aériennes, verticales et obliques, ou au sol, des plans techniques ${ }^{4} .$. répondant à des échelles variées, allant du lieu à la région. Certaines de ces sources sont par nature spatialisées, à l'image des cartes ou des plans; d'autres, notamment textuelles ou iconographiques, semblent ne pas se prêter spontanément à la 
représentation cartographique. Après un examen critique des sources, visant à ne retenir que celles qui offrent une fiabilité et une précision satisfaisantes (Valette et Carozza, 2010), le SIG historique va rendre possibles la visualisation et la superposition de ces informations d'époques et de natures différentes, et leur association dans le cadre d'études comparatives. Le géoréférencement des cartes et plans les replace à une échelle identique, facilitant leur comparaison. La localisation des documents iconographiques offre un regard sur les paysages sous un angle complémentaire. Quant au remplissage des tables associées, il rend possible la spatialisation de documents textuels, qui ne semblaient pas a priori cartographiables. Outil d'origine quantitative, le SIG est ainsi à même de gérer et d'intégrer des sources de données qualitatives, notamment écrites, élargissant les domaines de recherche et les possibilités offertes à ses utilisateurs (Gregory et Knowles, 2011). Il permet la confrontation entre eux des documents anciens et contemporains établis à diverses échelles, présentant des différences géométriques et thématiques notables (Franchomme et Dubois, 2010).

6 Ainsi, sur le site de Nancy, le SIG historique en cours de réalisation reconstitue l'avancée de la ville pas à pas à l'aide de plans anciens, mais également de sources d'archives telles que les listes nominatives ou le casier sanitaire ; on y note également l'évolution des bras de la Meurthe et des cours d'eau affluents, aujourd'hui disparus car tubés et enfouis, mais aussi la fluctuation des activités humaines liées à l'eau. L'onde de crue de 1947 - la plus importante connue à ce jour par la ville - a également pu y être reconstituée.

7 L'objectif de l'outil est ensuite double. D'une part, la production d'un recueil de cartes historiques représentant la situation paysagère et urbaine à dates régulières. Mais l'outil SIG prend toute sa dimension dans l'exploitation des renseignements des tables associées aux éléments cartographiés. Statistiques, graphiques... issus de l'analyse spatiale, ils offrent une approche quantitative agrégée à la lecture spatiale des paysages. D'autre part, au-delà de simples calculs et d'informations (superficies dédiées aux différents espaces, densités, répartition de populations ou d'activités...), l'outil facilite la mise en œuvre et la représentation cartographique d'analyses plus poussées, illustrant notamment les évolutions spatiotemporelles des territoires, ou les trajectoires empruntées par ces structures paysagères (Franchomme et Dubois, 2010).

Le regard géohistorique et rétrospectif sur la relation entre une ville et son cours d'eau éclaire les démarches opératoires qui conduisent à la production de la ville. Cette approche permet d'entreprendre des démarches concertées en lien avec le territoire et s'appuyant sur un récit (réel ou sublimé) qui permet de promouvoir les aménagements de demain (Sgard, 2008). Ces derniers ne constituent plus des ruptures opératoires brutales, mais accompagnent des processus de transition. Ils ont pour objectifs de justifier, d'éclairer et de concilier auprès des acteurs civils et institutionnels les choix d'aménagement.

\section{Histoire d'un couple : Nancy et la Meurthe}

9 Ville médiévale ${ }^{5}$, Nancy a été établie sur la première terrasse alluviale de la Meurthe (220-226 m), dans une cuvette dominée par les côtes de Moselle. La fondation politique de la capitale ducale reste liée à une médiocre fonction de franchissement, la ville s'implantant dans l'étranglement de la vallée entre le plateau de Maxéville et celui de 
Malzéville. Depuis cette fondation, la relation du couple ville-Meurthe s'est organisée en trois temps de durée inégale.

\section{Le temps de la défiance (des origines au milieu du XIX ${ }^{e}$ siècle)}

10 La ville pionnière domine une vallée occupée par une large zone humide (prairies, marécages et anciennes forêts alluviales), seul le pont de Malzéville permet de passer la rivière. Le franchissement est amélioré par la construction d'un solide ouvrage à treize arches en pierre. Cependant, malmené, l'ouvrage reste vulnérable aux crues et aux effets des conflits.

11 Malgré ce site de franchissement, la Meurthe est peu mobilisée par l'organisme urbain naissant. Cette fragilité du lien résulte de multiples raisons, d'interactions entre territoires, milieux et espaces, et de géopolitique régionale. En effet, la Meurthe ne répond pas aux trois principaux usages de l'époque :

- être un agent moteur d'une économie préindustrielle en favorisant une navigation fluviale et une activité portuaire génératrice de richesses; la Meurthe reste médiocrement flottable notamment en raison de ses étiages. L'activité de batellerie, à l'exception faite des trains de bois descendant de Raon-l'Étape jusqu'au Rhin à Coblence (Mathis et al., 2019), demeure modeste. Saint-Nicolas-de-Port a, bien avant Nancy, structuré le commerce, les ruptures de charge et les flux de la Meurthe;

- répondre aux usages domestiques: ses eaux légèrement salées sont impropres à la consommation, la ville a donc dû drainer les eaux des ruisseaux cataclinaux pour s'approvisionner en eau potable, développant étangs et « viviers " pour ses besoins ;

- offrir une mise en scène de cette relation: la Meurthe symbolise à l'époque le danger générant la défiance, par peur des crues ou des étiages, mais aussi par les difficultés d'assurer le franchissement et le rapprochement des rives; d'où le maintien de vastes étendues de prairies humides piquetées de bras morts tardivement conservés (Prêcheur, 1953).

12 Cette relation de défiance dure environ cinq siècles. Le lien du cours d'eau avec la cité est longtemps resté ténu, la ville tournant le dos à sa rivière. Pendant tout ce temps, l'hydromorphologie fonctionne comme une juxtaposition de deux systèmes. Dans la fabrique de la ville, la croissance urbaine de l'époque moderne matérialisée par la Ville-

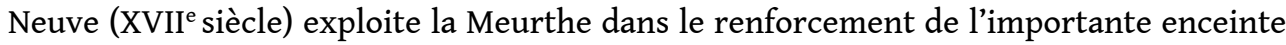
aux remparts polygonaux. La rivière et ses zones humides, mais aussi la fragilité des possibilités de franchissement, sont mobilisées tel un glacis et un hydrosystème défensif (Husson, 2018). La démilitarisation de la ville et l'effacement des remparts, faisant suite aux longues périodes d'occupation par les troupes françaises sous Louis $\mathrm{XIV}$, puis les extensions urbaines du XVIII ${ }^{\mathrm{e}}$ siècle (notamment la mise en chantier des lieux et marqueurs du nouveau pouvoir tels que la place Stanislas en 1754), se préoccupent bien peu de la rivière. Toutefois, la ville rêve d'aménager ces espaces rivulaires et dresse quelques projets non aboutis, tel celui d'un vaste "jardin d'eau » conçu par Jules Hardouin-Mansart (1703), ou encore celui du port et de son canal, pensé par l'hydraulicien François-Michel Lecreulx (1785). 
Figure 1. En 1835, la ville historique se défie de la Meurthe

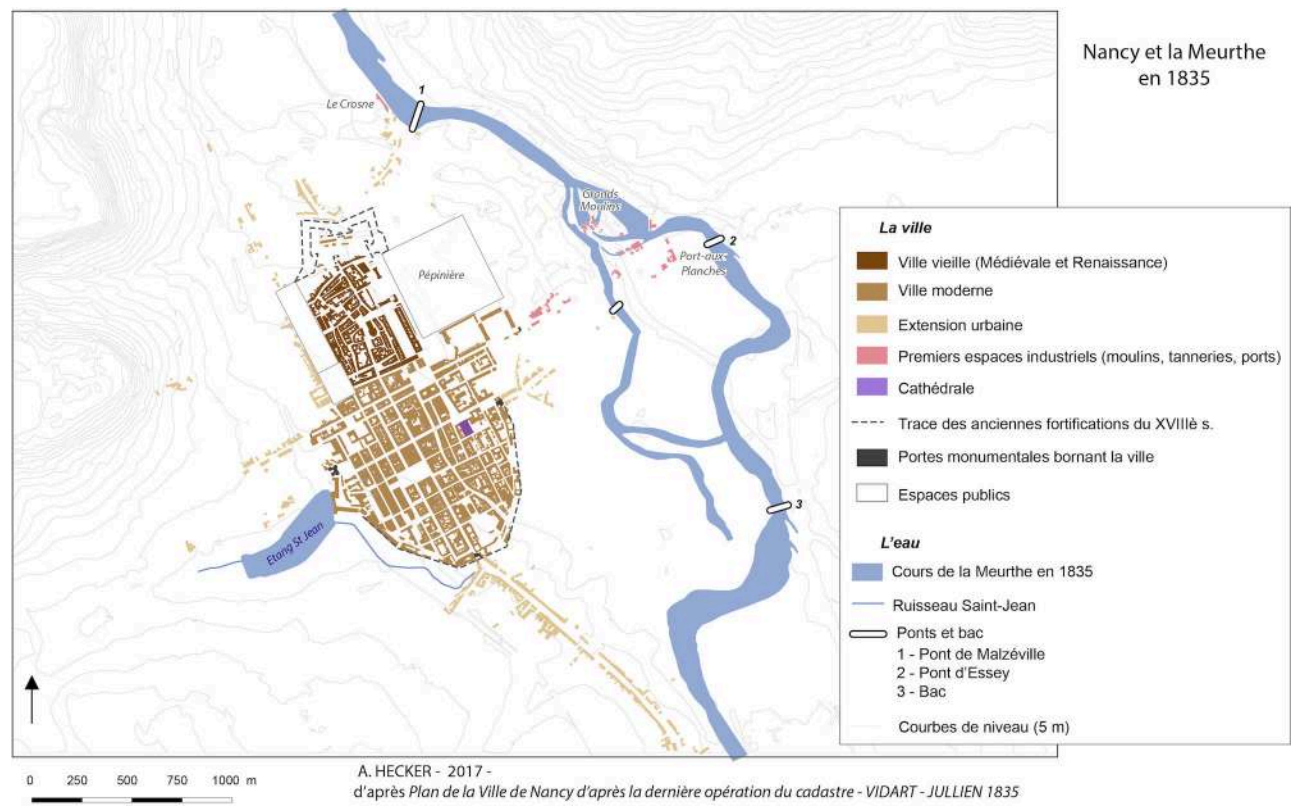

La carte souligne la timide extension vers le lit de la Meurthe. Les deux systèmes sont comme juxtaposés. Seuls deux ponts établis en 1498 (pont de Malzéville) et 1750 (pont d'Essey) relient la ville à la rive droite de la Meurthe. En amont, un bac dessert Tomblaine.

13 La faible croissance urbaine de Nancy ne pousse pas la ville à se rapprocher de la rivière. La porte monumentale Sainte-Catherine ${ }^{6}$, qui marque l'entrée de ville, et l'actuelle caserne Thiry, construite entre 1765 et 1769, n'illustrent qu'une timide extension vers la Meurthe.

\section{La confrontation (milieu XIXe siècle - 1982-1983)}

14 Comme le souligne le rendu des cartes SIG empilées, le XIXe siècle est un temps de confrontation entre la ville et le fleuve. Ainsi, après 1871, Nancy connaît un essor exceptionnel, sa population triplant en quarante ans, conséquence de la conjonction de l'essor industriel et du bénéfice né de la nouvelle position de la frontière dessinée par le traité de Francfort (1871) (Dion, 1974 ; Hecker, 2017b).

Cette croissance se traduit par une extension urbaine structurée par les nouveaux quartiers militaires au sud de la ville, mais aussi par un étalement désordonné, voire anarchique, typique d'une "ville-champignon", que la municipalité essaie tant bien que mal de structurer en déployant axes viaires et réseaux de transport (Hecker, 2017c). Cette poussée urbaine d'une modeste ville administrative, devenue temporairement "ville frontière ${ }^{7}$ ", s'accompagne d'un important développement des industries manufacturières, et notamment des usines sidérurgiques, entre les rives de la Meurthe et le canal, espaces favorables à ces activités mais très exposés aux crues, ainsi que dans les banlieues de Jarville, Maxéville, Pompey et Neuves-Maisons. Au-delà des terrasses non inondables, le lit majeur de la Meurthe est investi par les usines, les entrepôts, les triages, les quartiers ouvriers, mais sans disposer de plan d'ensemble, à l'image d'une ville grandie trop vite. La demande urbaine favorise l'extension des ceintures nourricières qui occupent les bonnes terres alluviales, notamment dans le secteur de Malzéville qui est colonisé par les maraîchers (De Gasperin et Mathis, 2017). 
Figure 2. La ville s'impose à la Meurthe

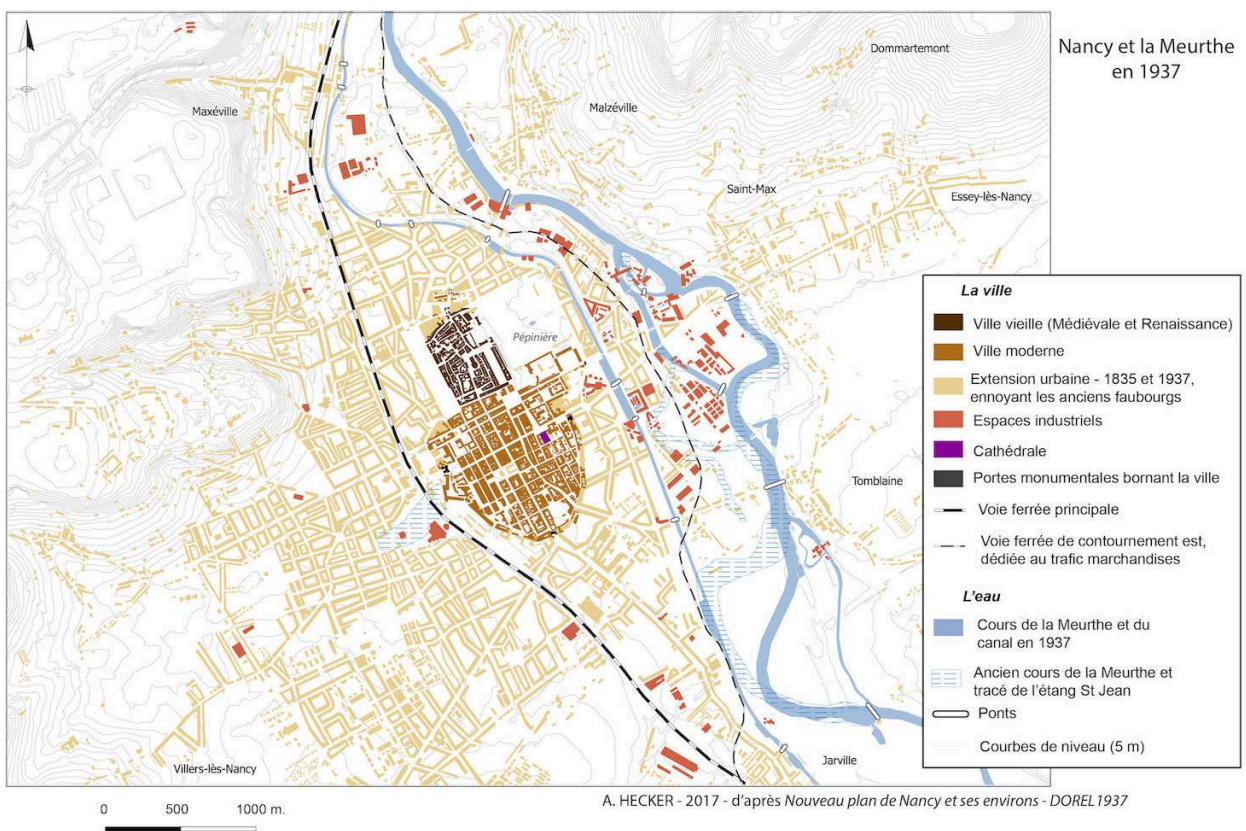

C'est le temps de la confrontation et de la conquête du lit de la Meurthe qui est détourné, linéarisé afin de répondre aux besoins de l'industrie sidérurgique et à la création de crassiers.

Cette phase de l'histoire urbaine conduit à la conquête de la vallée de la Meurthe. Elle contraint la mutation du cours d'eau, fixant progressivement le tracé de ce dernier, gommant les bras morts, les bancs de sable et les méandres, bloquant les divagations... La Meurthe est progressivement encorsetée par le tissu urbain et les infrastructures de transport. Cependant la rivière reste une menace. Les travaux d'aménagement et de domestication du cours ne parviennent pas à mettre la ville à l'abri des crues, comme l'illustrent celles, dévastatrices, de 1910 ou encore de 1947 (Nicod, 1949). 
Figure 3. Nancy et la Meurthe en 2017

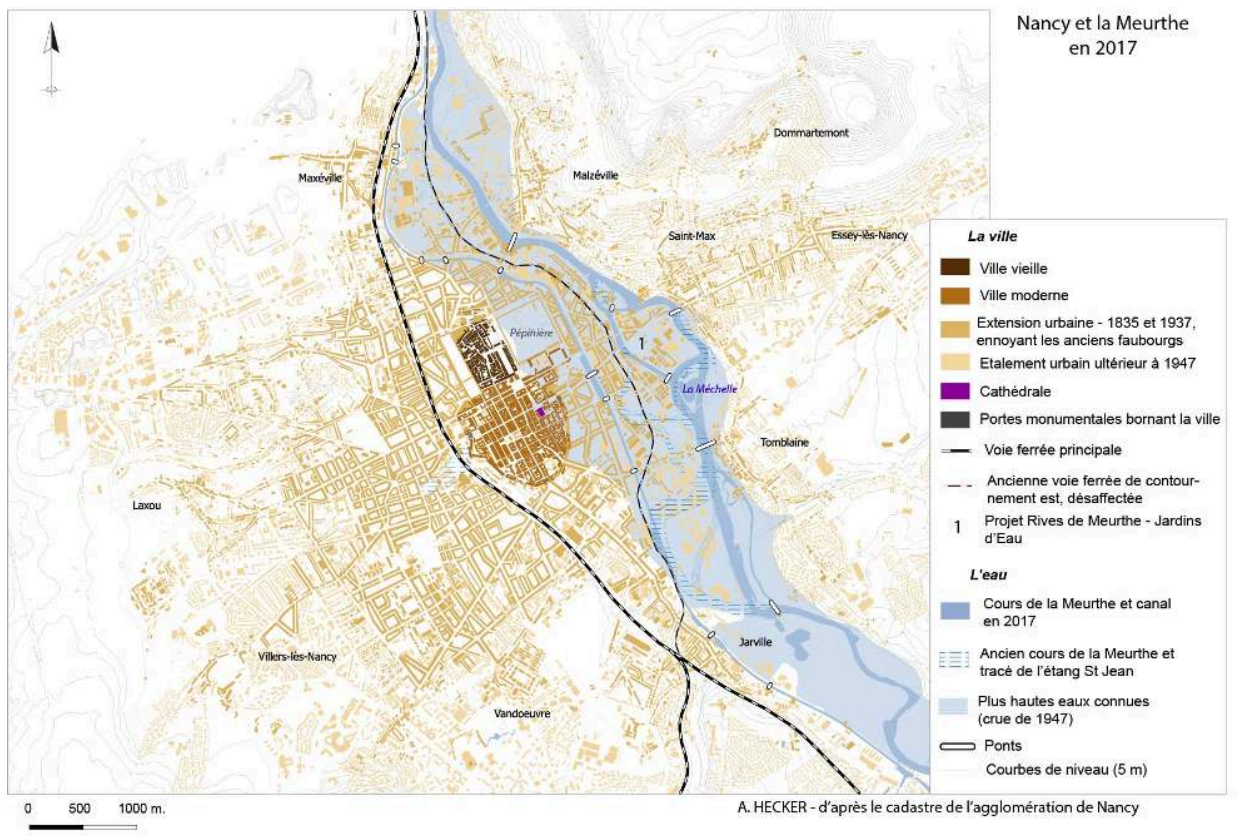

La vulnérabilité de la cuvette nancéienne oblige désormais les acteurs de l'aménagement à modifier la perception des paysages de la rivière. C'est le temps de la reconquête.

Le paysage né de cette croissance urbaine est segmenté par les tracés logistiques, notamment le canal de la Marne au Rhin et le diverticule ferroviaire de la gare des marchandises Saint-Georges. Il est complété par la Meurthe et ses sous-affluents, des lambeaux de jardins et de zones humides... La naissance de ces nouveaux quartiers ne crée pas véritablement de la ville, mais relève plutôt d'un processus de déversement de ce qui ne trouve pas place dans la cité. Si les grandes crues de 1910 ou de 1947 (centennales) sont restées dans les mémoires nancéiennes, une nouvelle rupture s'opère à la suite de celles, répétées, de 1982 et 1983. Ces dernières se produisent dans un contexte de désindustrialisation, déconstruisant usines, ateliers et hangars situés dans l'interstice Meurthe-canal (Edelblutte, 2006), conséquence de la transformation économique de Nancy (Dion, 1994). Ce processus clôt en partie la boucle de l'accident historique de la frontière. Il s'agit d'un « retournement » de l'histoire nancéienne qui met un terme au temps de la confrontation et annonce un temps de concertation qui se traduit par une reconnexion entre les deux systèmes qui se sont succédé. Ce retournement est rendu possible car, comme l'affirme Sophie Bonin (2007), « les friches ont dégagé du foncier et ouvert un espace de liberté. Elles fournissent l'occasion de repenser les fonctions économiques et sociales, voire l'identité de ce territoire ».

\section{La concertation/la reconnexion (1983 à nos jours)}

La vulnérabilité du quartier industriel, mais également celle de la ville moderne (XVII ${ }^{e}$ XVIII siècle), jugée admissible et faisant l'objet d'aménagements souvent empiriques jusqu'en 1982-1983, est désormais devenue inacceptable et insupportable. Aussi, 
l'épisode répétitif des crues qui ont frappé la ville amène à concevoir une politique d'aménagements destinée à réduire sa vulnérabilité face au cours d'eau, préalable à l'entame d'une reconquête urbaine. Ce processus est lent, lié à la faible dynamique démographique de l'agglomération nancéienne, et perturbé en 2012 par un nouvel épisode de crues, mettant au jour des enjeux inédits (Chiffre et al., 2014). Les riverains ont été informés et associés aux travaux menés. Ils ont pu en particulier bénéficier d'accès privatifs vers l'ancien chemin de halage devenu promenade.

\section{Diminuer la vulnérabilité en tenant compte de la rivière}

19 Les inondations ont en effet conduit à mener des opérations d'aménagement/ ménagement, à même de réconcilier progressivement le couple ville-rivière. De 1986 à 2002, les travaux financés par la communauté urbaine du Grand Nancy (CUGN), l'État, la région Lorraine et le département de Meurthe-et-Moselle couvrent onze kilomètres de rives distribuées en quatre sections. Les tranches de travaux à engager sont retenues en établissant une grille de priorités. Elles sont déclinées en tenant compte des spécificités de l'hydrosystème local : le faible débit de la Meurthe $\left(41 \mathrm{~m}^{3} / \mathrm{s}\right.$.), et la présence des bras anthropisés hérités des implantations industrielles et du tracé du canal à petit gabarit de la Marne au Rhin. Entre 1986 et aujourd'hui, Nancy retisse ses liens avec la Meurthe au gré des urgences qu'il fallait faire aboutir.

20 Le génie civil adapté au contexte meurthois a conduit à enrocher, endiguer, corseter, poldériser l'aval qui court de la confluence avec la Moselle pour s'arrêter sur le nouveau barrage des Grands-Moulins. Tous les types de travaux sont mis en œuvre: construction de seuils, recalibrage du lit mineur, construction de murs/digues bordant les quais, et aménagement d'un bras de décharge.

21 Les travaux de recalibrage et de reprofilage de la rivière ont nécessité la construction d'un barrage à clapets modulables, capable d'évacuer une crue de $800 \mathrm{~m}^{3} / \mathrm{s}$. Essentiels pour se prémunir des effets des crues, ces travaux n'ont toutefois que peu tenu compte de la respiration et de la liberté à accorder à la rivière, dans cette partie en aval de la traversée de l'agglomération. Cependant, au fur et à mesure des travaux décidés en remontant le cours de l'aval vers l'amont en traversant l'agglomération, les projets de rapprochement de la ville et de la rivière évoluent et soulignent une transition entre morphologie urbaine conquérante et cité interagissant avec son milieu. Ils sont réfléchis à trois échelles, celle de la ville, celle de l'agglomération avec la continuité attendue jusqu'à la confluence avec la Moselle à Frouard-Ban-la-Dame, et celle de l'enveloppe métropolitaine, voire du Sillon lorrain.

À partir de 2005, l'approche opératoire conforte la respiration de la rivière. Elle mobilise une représentation $\mathrm{du}$ paysage naturel $\mathrm{du}$ cours d'eau. Le projet de réaménagement concède de la place aux plages d'étale des eaux qui existaient en amont, là où avaient été préservés des prairies humides, des marais et des roselières. Ce processus a été particulièrement développé lors de la dernière tranche de travaux. Celle-ci tient compte également de la très forte croissance de population, qui déborde de la cuvette nancéienne pour se déverser au sud de la ville, de l'artificialisation des sols et du risque d'inondation par ruissellement aggravé par la configuration en amphithéâtre dominé par la côte du Bajocien. Ainsi, un bassin de rétention de 4,4 ha et de $40000 \mathrm{~m} 3$ est créé en 2011 pour recueillir en amont de Nancy les eaux des ruisseaux de l'Embanie et du Flahaut. Ce bassin complète également les aménagements sur la Meurthe car il doit permettre d'écrêter ses crues. Ces travaux s'accompagnent de la 
restauration du cours de la Meurthe de la section de l'Hurpont, soumis à une importante érosion. Lors de leur achèvement, ces travaux ont été présentés comme un modèle de qualité paysagère et de restauration de la biodiversité.

\section{Reconquérir la ville et la reconnecter à la Meurthe}

La respiration et la mise en scène des paysages urbains rivulaires sont exprimées dans les opérations de renouvellement urbain (Husson, 2019). Cette mise en miroir de la ville en direction de la Meurthe est marquée par la réalisation des «jardins d'eau », le long du canal, face au port Sainte-Catherine, préalable d'une vaste opération de rénovation, de restauration et de constructions. Ce "motif urbain", réalisé par le paysagiste Alexandre Chemetoff sur le nouvel espace «Rives de Meurthe » et désormais dupliqué dans la ville ${ }^{8}$, constitue une ré-union, qui évolue avec l'ambition de créer une ville structurée par une trame bleue. "La symbolique des "Jardins d'eau » illustre cette domestication de l'eau et témoigne du renouveau du quartier. L'eau en devient désormais l'élément identitaire.» (Chiffre et al., 2014). Cependant, tenant compte du contexte et de la mémoire de l'inondation à Nancy, «l'opération Meurthe-Canal ouvre enfin la ville vers l'eau - en réalité plus vers le canal que vers la rivière proprement dite - lui offrant enfin ce waterfront, espace "nautique" de promenade, de plaisance, de sports et de loisirs dont elle avait toujours historiquement manqué » (Edelblutte, 2006).

Figure 4. L'hydrosystème meurthois dans la ville (vue nord-sud, soit de l'aval vers l'amont)

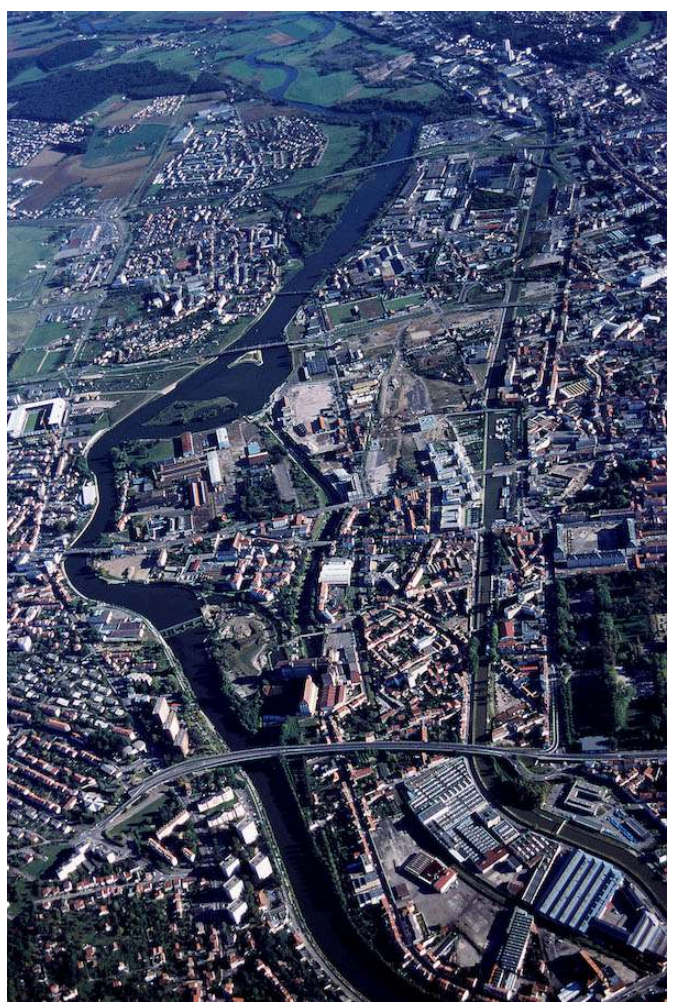

Avec au premier plan le quartier industriel du Crône puis, plus en amont, la division de la rivière entre plusieurs bras et le tracé rectiligne du canal de la Marne au Rhin ; enfin, en haut de l'image, la plaine de Tomblaine et les espaces de respiration conservés pour étaler des crues. Cette photographie prise d'aval en amont illustre les démarches opératoires de plus en plus protectrices et environnementales menées sur le cours de la Meurthe.

Photographie : Cerpa - André Humbert, Colette Renard-Grandmontagne. 
Figure 5. La transformation de l'ancien quartier industriel en lien avec l'aménagement du lit de la Meurthe

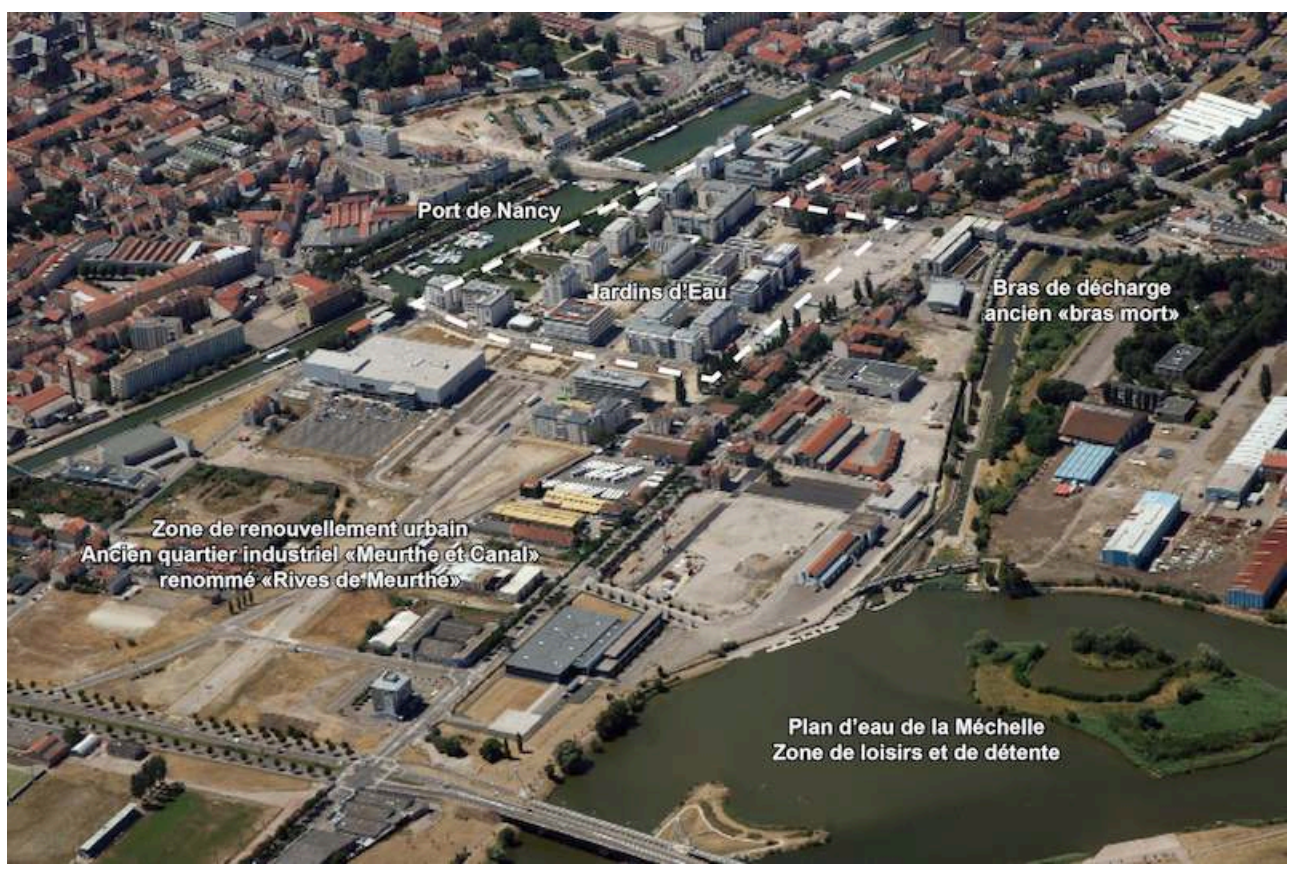

Une première étape dans la régénération urbaine des «Rives de Meurthe ».

Photographie : Cerpa - André Humbert, Colette Renard-Grandmontagne.

Figure 6. Croquis modélisé de la reconquête urbaine et de l'approche paysagère de l'espace « Rives de Meurthe"

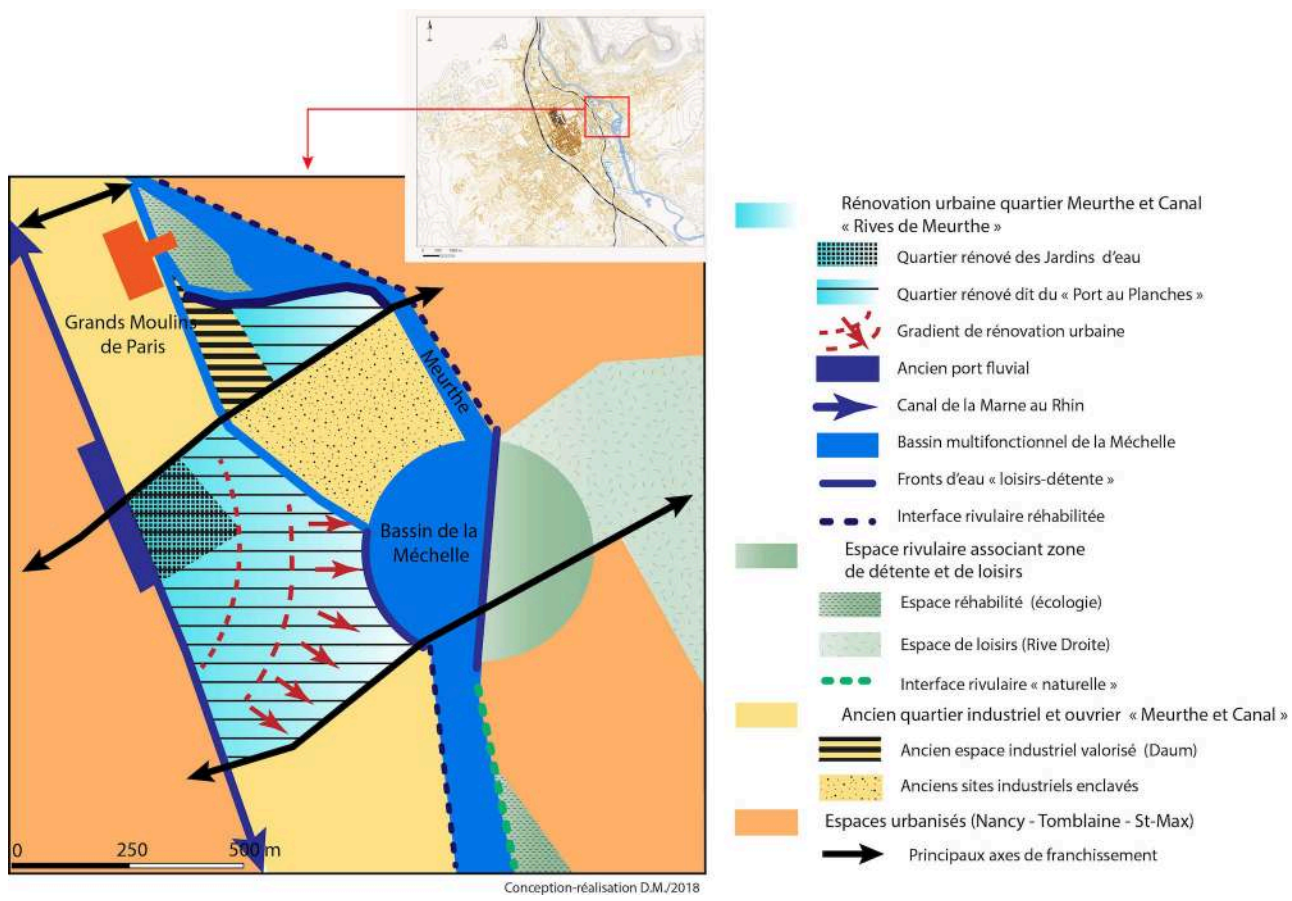

Depuis cette première phase de la reconquête, les travaux de renouvellement urbain se poursuivent. Ils associent de plus en plus la rivière perçue comme un patrimoine du quartier, ce qui est conforté par l'absence d'impact de la crue de 2006. Ainsi l'approche 
opératoire, le réaménagement paysager, le river landscape confortent la nature et la respiration de la rivière, un front d'eau se construit, fondé sur les loisirs et les espaces de détente. La priorité est accordée à la promenade, à la conservation de jardins potagers, à la préservation des ripisylves. Cette inflexion au profit de la renaturation n'interdit pas de jeter sur la rivière le vaste pont suspendu de la Concorde et sa passerelle, destinés à mieux s'affranchir des ruptures entre la ville et sa rive droite. Ainsi comme le décrit Sylvie Salles (2011) : «La Meurthe [...] est devenue un espace de la ville dont les qualités esthétiques et écologiques s'apprécient dans la pratique des berges ou du cours d'eau. C'est en tant que paysage "naturel" que l'environnement de la rivière est perçu. »

\section{Des nouveaux enjeux, une diffusion des « paysages d'eau » dans la ville}

L'analyse des inondations du 21 mai 2012 a permis de compléter et de diffuser cette approche dans la ville. Cet aléa climatique exceptionnel, conséquence de deux orages successifs ayant déversé $112 \mathrm{~mm}$ de précipitations cumulées en 3 heures à Essey-lèsNancy (soit au total, 11 millions de $\mathrm{m}^{3}$ pour l'ensemble de la cuvette nancéienne), a révélé de nouvelles vulnérabilités liées aux inondations par ruissellement et la nécessité de tenir compte du réseau hydrographique secondaire dans la gestion globale des risques (Chiffre et al., 2014). La densification de l'Est de l'agglomération nancéienne, qui conduit à l'essor de la zone d'activités de la "Porte verte ", a souligné les enjeux entre l'artificialisation des terrains de la rive droite et le risque d'inondation, et le débordement $\mathrm{du}$ cours $\mathrm{du}$ Grémillon. Ces aspects avaient été négligés dans l'aménagement et les opérations de rénovation urbaine: quartier de Jartom à Tomblaine dans le cadre de l'Agence nationale de rénovation urbaine (ANRU), aménagement de la zone de Mouzimpré, requalification de l'ancienne caserne Kléber à Essey-lès-Nancy... (Mathis, 2012). Une phase de réflexion et d'adaptation du réaménagement de la rive droite s'est opérée à la suite de ces événements, afin de réintégrer ce cours d'eau au sein du tissu urbain. Opérés en 2016-2018, les travaux de restauration partielle du ruisseau, en suivant les concepts de l'ingénierie de la restauration des cours d'eau, façonnent le Grémillon suivant les principes d'un river design pour produire un river landscape (Prominski et al., 2012; Lespez et Germaine, 2016). Cet aménagement qui prolonge les travaux de l'ANRU (convention signée en 2007) conforte une reconquête fondée sur une construction paysagère développant un aspect "plus naturel». Ainsi, comme l'affirme Aurélie Michel (2012): "La greffe transposée à l'imaginaire urbain fait naître une configuration singulière de l'habitat. Il s'agit alors d'effectuer un transfert intelligent de morceaux de nature à l'intérieur de la ville, opérant un renouveau de cette dernière grâce aux structures végétalisées, qui se développent selon un principe de régénération de l'espace.» Pontons, plantations jardinées, mobilier urbain établissent de nouvelles "aménités " qui intègrent un paysage «renaturé» du cours d'eau et participent à la reconquête d'espaces marginalisés. C'est à l'échelle du réseau hydrographique secondaire que s'opère la reconquête de la ville avec la Meurthe et son canal. 


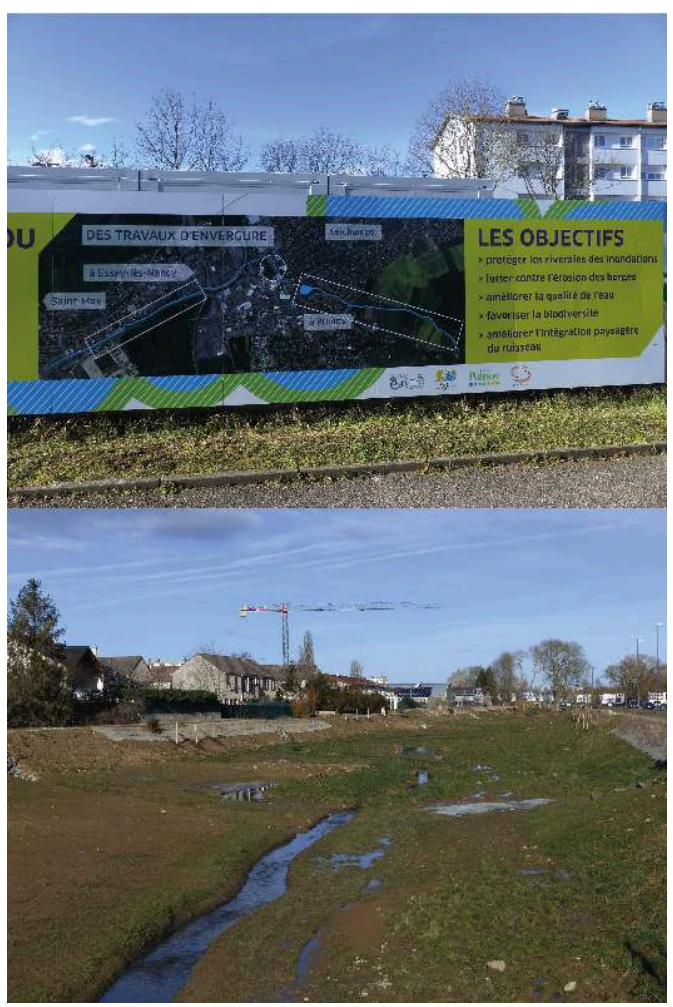

Les travaux ont transformé le cours du ruisseau en requalifiant le paysage rivulaire par des plantations, des talutages, un reméandrage...

Photographies : Denis Mathis.

La reconquête de l'espace rivulaire a progressé et s'est diffusée depuis les jardins d'eau et le bassin de la Méchelle dans l'agglomération et dans la vallée de la Meurthe. Les démarches appliquées ont évolué progressivement. Désormais, elles tiennent compte d'une approche présentée comme plus respectueuse de l'environnement. Elles intègrent à la réflexion opératoire une représentation de la nature dans le respect d'un hydrosystème dynamique. Cette dernière retrouve une place au sein du tissu urbain (Lechner, 2006). Cette approche traduit un projet de territoire qui vise à construire une trame bleue dans une démarche volontariste, intégrée à une démarche de renouvellement urbain. Les vieux quartiers industriels et leurs «traces » paysagères constituaient pour la ville un marquage territorial en creux. En bordure d'un espace urbain déclassé, la Meurthe était rendue responsable de la désaffection et de la désindustrialisation du quartier ouvrier. Désormais cet espace interstitiel compris entre Meurthe et canal constitue un noyau de reconquête, créant un péricentre ouvert sur la Meurthe dans une lecture duale de deux fronts d'eau : un front résidentiel tourné vers le canal et un front de loisirs, de détente et de nature tourné vers la Meurthe. Ce modèle est destiné à être dupliqué pour favoriser la requalification des espaces industriels désaffectés au nord et au sud de Nancy (Grands-Moulins de Paris, site de l'usine la Rochette 9 à Jarville...). La question de l'hydromorphologie nancéienne traduit un itinéraire historique et spatial qui cherche à assurer une relation apaisée entre la ville et la rivière. Elle souligne un changement complet de paradigme et construit un nouveau récit afin de soutenir les récentes opérations d'aménagement justifiant 
l'affirmation de Sylvain Rode: "La reconquête des cours d'eau urbains semble donc avant tout servir des objectifs d'aménagement urbain » (2017).

\section{Contrastes et analogies des dynamiques de reconquête paysagère des cours d'eau au sein des couples villes-fleuves du Sillon lorrain : les cas de Metz et Thionville}

Dans le Sillon lorrain, la question du rapport de la ville au cours d'eau est liée aux enjeux de la vulnérabilité des villes face aux risques d'inondation. Bien que l'hydrosystème de la Moselle ${ }^{10}$ s'observe à une autre échelle que celui de la Meurthe, étudier les relations et les paysages des couples villes-rivières à Metz et Thionville permet de constater les similitudes et les divergences dans l'approche de ces relations.

\section{Metz et la Moselle, un couple bien établi}

Depuis la colline Sainte-Croix, Metz surplombe la confluence de la Seille et de la Moselle, et la conquête des rives y a été plus précoce qu'à Nancy. Le couple villeMoselle est mieux établi car la Moselle, dès l'origine, est un agent générateur de richesses pour la république marchande, et ce malgré ses crues dévastatrices. Dès l'époque médiévale, de nombreux ponts relient la rive droite aux îles et à la rive gauche, et à son important vignoble. La batellerie est active sur la Moselle, mais aussi sur la Seille jusqu'à son déclin et à son abandon au XVI siècle.

Les crues de la Moselle sont aussi marquées qu'à Nancy. À Metz, elles ont balayé régulièrement les îles de la Moselle (Saint-Symphorien, Saulcy, Chambière...). L'approche géohistorique permet de reconstruire une mémoire de la crue avec des informations d'abord qualitatives (Ferber, 2012). Ces dernières servent à établir une chronologie, notamment pour des épisodes exceptionnels récurrents $(1224,1315,1373$, $1415,1421,1427,1483,1631,1778,1804,1870,1939,1942 \ldots)$. L'inondation de 1421 est particulièrement remarquable puisqu'elle submerge les grands ouvrages du pont des Morts et le quartier du Pontiffroy, fournissant une précieuse indication concernant le volume de la crue. Cependant comme à Nancy, la crue de référence reste celle centennale de 1947 et celles de 1982-1983.

Cette menace, représentée par les crues de la Moselle et de la Seille ${ }^{11}$, a été longtemps acceptée en raison des avantages qu'apportent les deux rivières. On observe cependant des aménagements empiriques tels que la digue de Wadrineau au Ban-Saint-Martin. Construite à l'origine en bois, elle fut rebâtie et consolidée au XVe siècle avec les débris des pierres de l'abbaye de Saint-Martin-lès-Metz. Elle couvre 320 mètres de berges avec une hauteur de remblais de $6,50 \mathrm{~m}$.

La densification de l'espace insulaire (quartier d'Outre-Moselle) et la militarisation au XVIII ${ }^{e}$ siècle avec la création du fort Moselle, des casernes de l'île Chambière puis, au $\mathrm{XIX}^{\mathrm{e}}$ siècle, de l'île du Saulcy, exposent les nouveaux quartiers au risque d'inondation. Aussi, à partir de 1727, cette urbanisation se double des travaux d'aménagement destinés à atténuer l'effet des crues. Ils sont entrepris et dirigés par l'ingénieur militaire Louis de Cormontaigne (1695-1752), et commandés par le maréchal de BelleIsle (1684-1761), gouverneur de Metz. Le génie militaire conduit une politique 
opératoire plus globale et moins empirique que précédemment. Il cherche à protéger les nouveaux quartiers établis le long des rives de la Moselle.

Les crues de 1982-1983 ont été pour Metz des moments de transformation du couple ville-rivière comme nous l'avons vu à Nancy. Le processus de démilitarisation à Metz a été comparable à la désindustrialisation nancéienne. Il s'agit d'un "déménagement » du territoire qui offre une opportunité de reconquête rivulaire et insulaire. Elle a libéré partiellement les îles permettant de reconstruire un paysage rivulaire concentrique avec, au cœur de la morphologie de l'espace insulaire, un bassin de loisirs qui s'est ramifié entre les multiples bras de la Moselle.

Figure 8. L'étalement en vert et bleu de Metz, là où perdurèrent très longtemps un hydrosystème de défense et un port aux planches

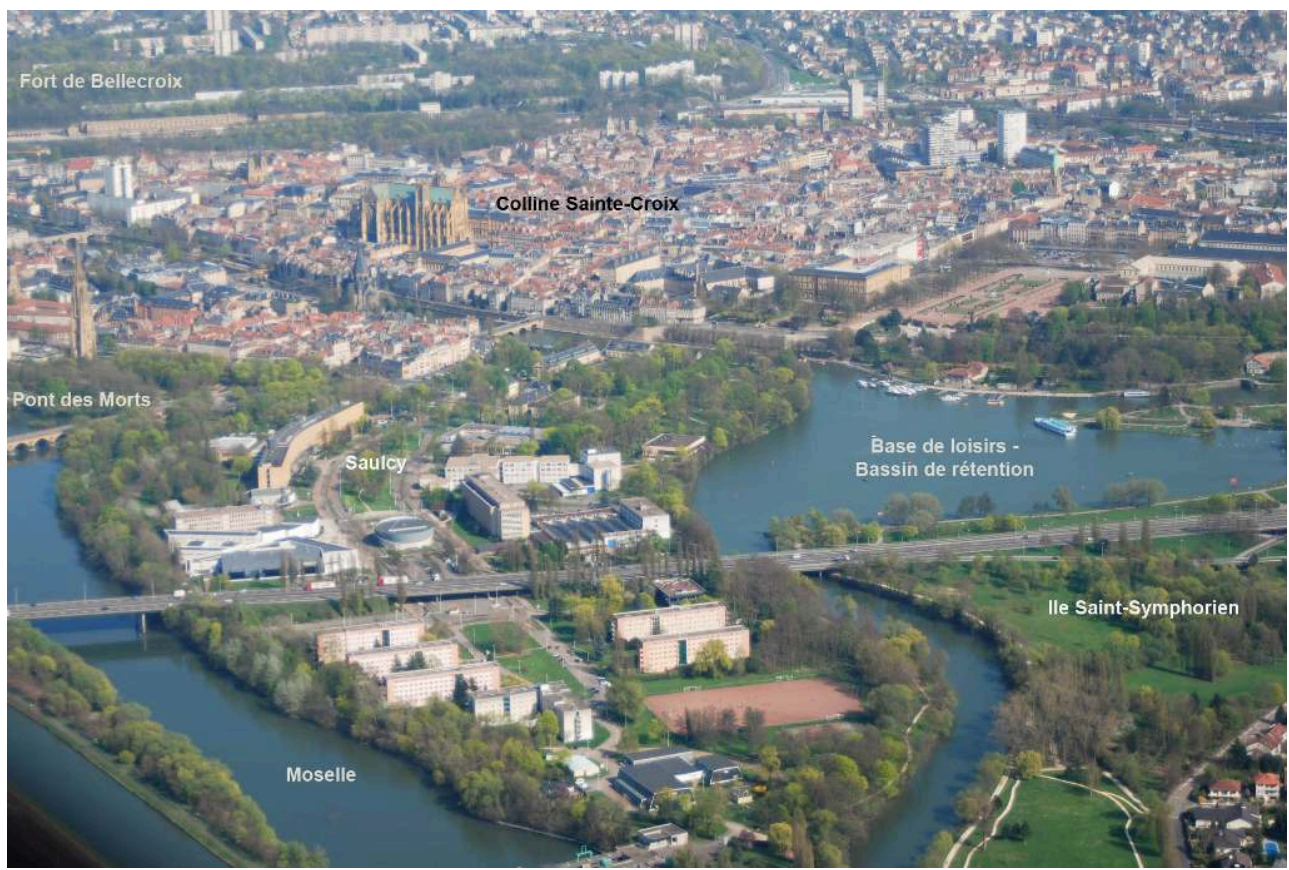

Lîle du Saulcy est investie par le campus qui jouxte le bassin de rétention des eaux. Au second plan, l'oppidum de Sainte-Croix, site initial de la ville gallo-romaine.

Photographie : Cerpa - André Humbert, Colette Renard-Grandmontagne. 
Figure 9.

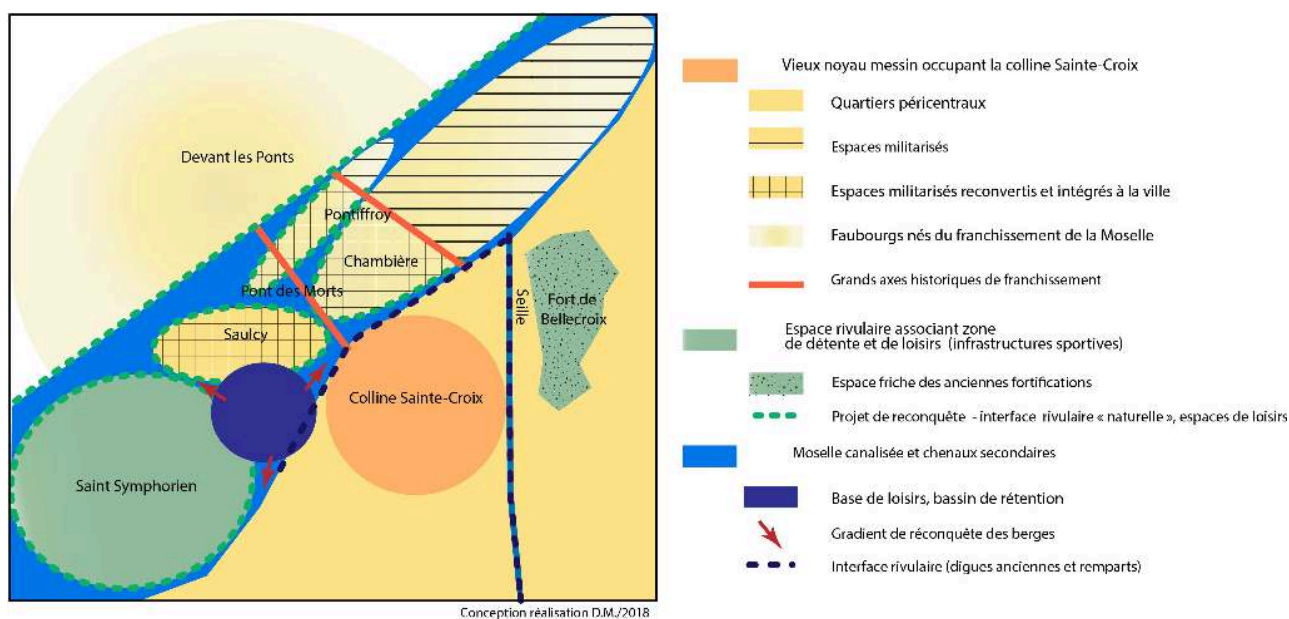

Les îles conquises lors de la militarisation sont aujourd'hui un enjeu de reconquête urbaine d'un front d'eau historique déjà bien établi.

Comme à Nancy, c'est à partir de ce noyau de reconquête que s'organise aujourd'hui la diffusion d'un nouveau rapport à la rivière. En 2016, Metz a mis en œuvre les premières étapes d'une mise en scène du paysage des berges des trois îles du Petit Saulcy, du Grand Saulcy et de l'île Chambière. Cette réappropriation/revalorisation des berges de la Moselle participe à la volonté d'associer davantage la ville à sa rivière.

\section{Thionville, une reconquête qui lie rive gauche et rive droite de la Moselle}

La situation thionvilloise est plus comparable à celle de Nancy qu'à celle de Metz, les deux villes se défiant du fleuve et de ses crues dévastatrices. La ville fortifiée de Thionville s'est d'abord adossée au fleuve, essayant de se préserver des crues de la Moselle. Elle se lie à la rive droite lors des aménagements militaires du couronné de

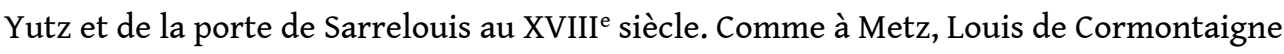
intervient, et fait creuser le canal de dérivation verrouillé par deux ponts-écluses fortifiés (1746), destinés d'abord à réguler le débit en protégeant la ville des inondations. Le noyau urbain est inscrit au sein d'une importante enceinte polygonale qui se déploie désormais de part et d'autre de la Moselle, assurant le contrôle et le franchissement du fleuve. Ces aménagements créent artificiellement une île qui sera le réceptacle des infrastructures de transport au XIX ${ }^{\mathrm{e}}$ siècle. 
Figure 10. Le paysage de l'agglomération thionvilloise conserve sa dissymétrie initiale

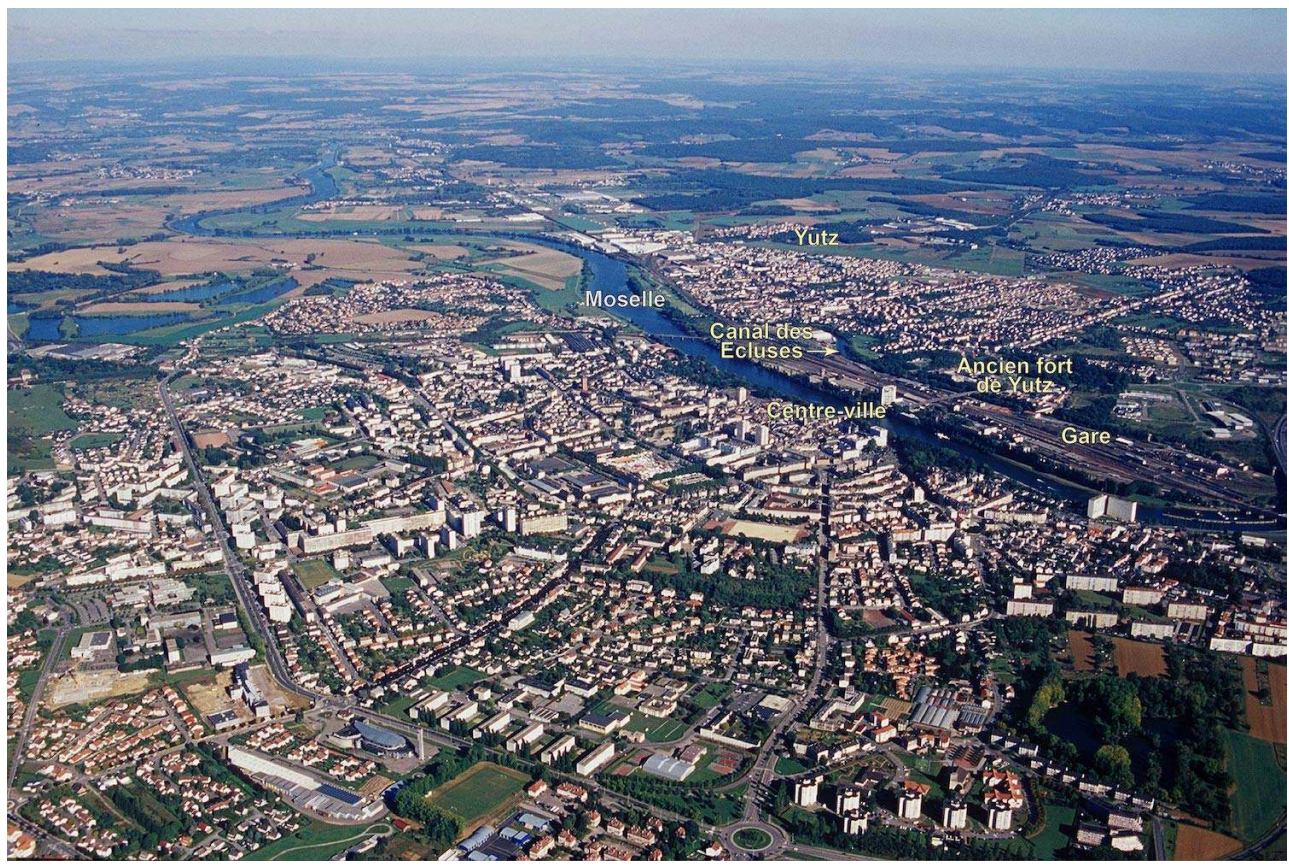

Le vieux noyau urbain sur la rive gauche de la Moselle s'étale en liaison avec la conurbation du Val de Fensch. Les emprises des infrastructures de transport (gare) et de l'armée (couronné de Yutz) multiplient les ruptures avec la ville de Yutz à l'arrière-plan.

Photographie : Cerpa - André Humbert, Colette Renard-Grandmontagne.

35 À Thionville, les enjeux de reconquête urbaine sont plus actuels qu'à Nancy et à Metz. Certes, les crues de 1982-1983 ont fortement marqué l'ancienne capitale du fer. Mais la crise industrielle et la décroissance urbaine ont fragilisé Thionville et y ont retardé la politique de reconquête, qui s'opère aujourd'hui depuis l'îlot de la gare. Ce quartier est en pleine redéfinition, s'organisant dans la gestion des flux pendulaires vers le Luxembourg et les dynamiques transfrontalières. Cependant, depuis ce quartier en cours de requalification, une impulsion nouvelle est mise en œuvre, enjambant le canal des écluses en direction de l'ancien fort de Yutz. Il s'agit d'une reconquête des anciens espaces militaires et industriels délaissés et marginalisés. 
Figure 11.

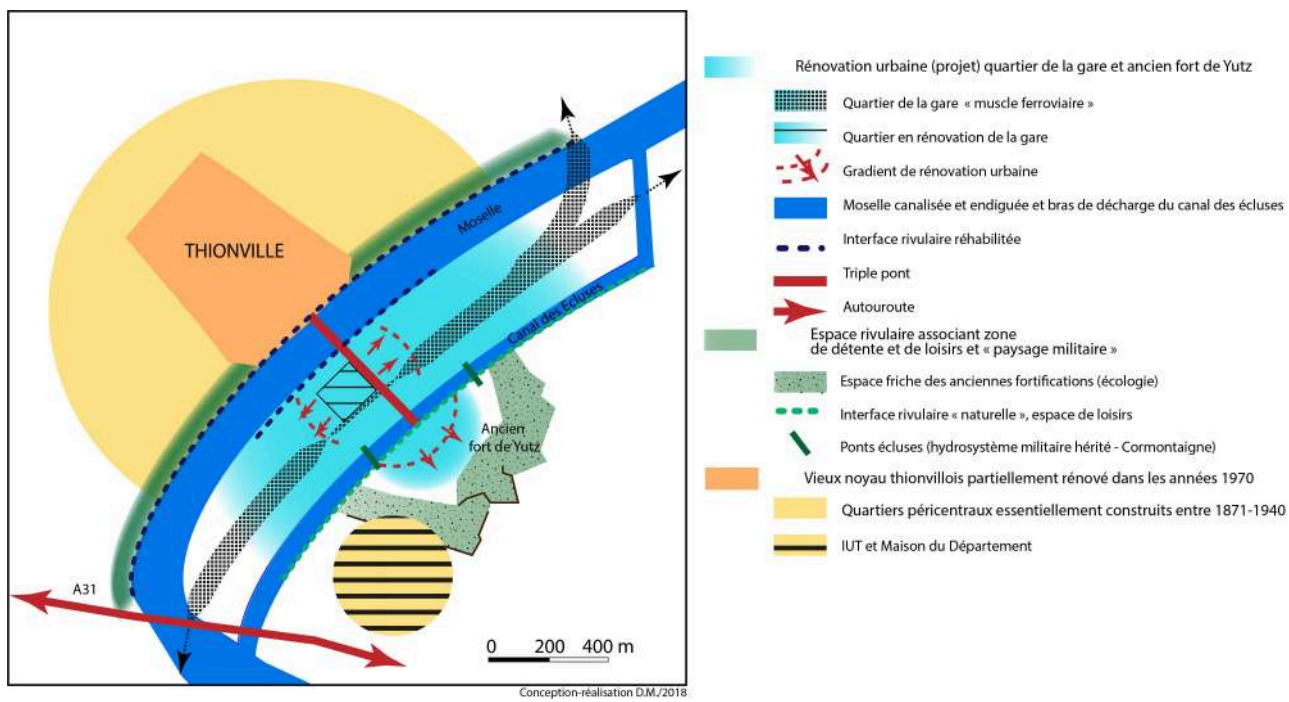

Les projets de requalification et de rénovation urbaine de l'agglomération thionvilloise passent par la reconquête du canal des écluses et des berges de la Moselle.

Cette phase opératoire doit répondre aux enjeux de la croissance urbaine en lien avec les phénomènes transfrontaliers. La friche militaire inscrite dans une renaturation spontanée, notamment le long du chenal des écluses, offre un cadre naturel déjà exploité par les loisirs (voies vertes). La gentrification des nouveaux espaces réhabilités induit une production paysagère à forte valeur environnementale et doit intégrer le canal des écluses, et se diffuser au sud de Thionville vers la commune de Yutz. Elle doit s'opérer également au sud-ouest en direction des friches industrielles de la sidérurgie (port minéralier d'Illange, Uckange...) que doit valider le projet de fusion des communautés d'agglomération de Thionville-Porte de France et du Val de Fensch. Cette mutation en germe devrait générer une nouvelle dynamique pour Thionville. Le processus de gentrification transfrontalière et de reconquête urbaine implique la construction d'un waterfront, établi de part et d'autre de la Moselle.

Dans les trois paysages des trois villes du Sillon lorrain que nous venons de présenter, comme le souligne Sophie Bonin en 2007: "Les opérations les plus exemplaires de reconquête des cours d'eau, au nom du paysage notamment (mais pas seulement), sont le fait des villes, dans un mouvement qui apparaît comme généralisé, se déroulant au même moment dans beaucoup des grandes villes riveraines, d'Europe. »

\section{Conclusion}

La connaissance géohistorique des couples ville-fleuve, ses aménagements, ses usages, ses conflits, la familiarité avec l'hydromorphologie urbaine et les paysages qui en résultent doit permettre d'éclairer et de souligner des siècles d'aménagements et de rapports équilibrés ou conflictuels face aux risques d'inondation. Le recours à la construction d'un SIG historique permet de tenir compte du processus de fabrique de la ville afin de reconstruire cette relation plus rationnellement que par le passé, surtout lorsque les informations historiques se révèlent empiriques et ne permettent pas une observation globale des paysages ou des aménagements. Ce recours à la géohistoire permet également aujourd'hui d'établir un récit qu'il est possible d'analyser et de 
comparer, afin d'observer comment les villes ont intégré cette gestion des cours d'eau. Bien que d'une manière opératoire, les politiques urbaines cherchent à concilier le patrimoine naturel aux démarches d'aménagement et aboutissent à la production de fronts d'eau, les récits locaux à Nancy, Metz ou Thionville ne sont pas identiques, les contextes évoluent. Aussi si Franck Scherrer affirme que «les politiques urbaines de renaturation s'inscrivent dans un même récit qui décode et recode le rapport de la ville à son environnement ", ces politiques doivent tenir compte des disparités des histoires urbaines. Le rapport à ces récits se modifie également avec le temps. En 2004, le contexte nancéien est différent de celui de 1983, et le récit opératoire destiné à tisserretisser les liens entre le cours d'eau et la ville intègre ce contexte. Revisiter cette cohabitation des couples villes-rivières traduite sur un temps long permet de reconstruire ces évolutions dans la vie du couple et d'établir une réflexion sur le futur de la relation ville-rivière.

\section{BIBLIOGRAPHIE}

Amoros, C., Petts, G.-E., Hydrosystèmes fluviaux, Paris, Masson, coll. d'écologie, n² 24, 1993, 300 p.

Bonin, S., «Fleuves en ville : enjeux écologiques et projets urbains », Strates, n 13, 2007, mis en ligne en octobre 2008, URL : http://journals.openedition.org/strates/5963.

Chiffre, E., Mathis, D., « Géohistoire militaire des étangs en Moselle », dans Touchart, L., Bartout, P. et Motchalova, O., (dir.), Mieux comprendre les étangs. Expériences nationales et internationales, Brive, Éditions Les Monédières, 2015, p. 398-401.

Chiffre, E., Mathis, D., Mathis, A., « Les inondations à Nancy. Anciennes et nouvelles problématiques ", Développement durable et territoires, vol. 5, $\mathrm{n}^{\circ} 3$, mis en ligne en décembre 2014, URL : URL : http://journals.openedition.org/developpementdurable/10665, DOI : 10.4000/ developpementdurable.10665.

De Gasperin, A., Mathis, D., « Malzéville : du rural à l'urbain, évolution spatio-chronologique d'une ville en périphérie de l'agglomération nancéienne », dans Masson, P., (dir), Malzéville, Histoire, paysage \& patrimoine, Haroué, Éditions Gérard Louis, 2017,141 p, p. 9-22.

Dion, R.-M., « De 1962 à 1992 : évolution des fonctions de la région urbaine de Nancy », Revue géographique de l'Est, t. 34, n² 2, 1994, p. 69-100, URL : www.persee.fr/doc/ rgest_0035-3213_1994_num_34_2_2271, DOI : https://doi.org/10.3406/rgest.1994.2271.

Dion, R.-M., «Effets des processus volontaristes dans la formation d'une région urbaine : Nancy et les plans d'aménagement et d'extension dans la première moitié du XXe siècle », Revue géographique de l'Est, t. 14, nos 3-4, juillet-décembre 1974, p. 245-311, URL : www.persee.fr/doc/ rgest_0035-3213_1974_num_14_3_1292, DOI : https://doi.org/10.3406/rgest.1974.1292.

Edelblutte, S., « Renouvellement urbain et quartiers industriels anciens : l'exemple du quartier Rives de Meurthe/Meurthe-Canal dans l'agglomération de Nancy ", Revue géographique de l'Est, vol. 46/3-4, 2006, mis en ligne en décembre 2009, URL : http://journals.openedition.org/rge/ 1455. 
Ferber, F., " Metz et ses rivières à la fin du Moyen Âge ", thèse de doctorat, université de Lorraine, Nancy, 2012, 810 p.

Franchomme, M., Dubois, J.-J., « Documenter les zones humides : vers une meilleure compréhension des paysages d'eau du XIXe au XXe s », Géocarrefour, vol. 85/1, 2010, p. 7-16, mis en ligne en juin 2010, URL : http://journals.openedition.org/geocarrefour/7622, DOI : 10.4000/ geocarrefour.7622.

Gregory I. N., Knowles A. K., 2011, « Using Historical GIS to understand Space and Time in the Social, Behavioural and Economic Sciences: A White Paper for the NSF ", Future Research in the Social, Behavioral \& Economic Sciences, janvier 2011, URL : https://eprints.lancs.ac.uk/id/eprint/ 39650/1/Gregory_Ian_78.pdf.

Guillerme, A., Le Temps de l'eau. La cité, l'eau et les techniques, Seyssel, Champ Vallon, 1997, 260 p.

Hecker, A., « Infrastructures et espaces urbains en transition », Revue géographique de l'Est, vol. 57,

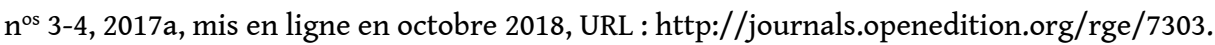

Hecker, A., « Les tramways historiques ont-ils "fait la ville" ? Le cas de Nancy ", Géotransports, $n^{\circ}$ 9, 2017b, URL : http://geotransports.fr/Revue/n09/pp_5-22_Les_tramways_historiques_ontils_fait_la_ville_Le_cas_de_Nancy_A_Hecker.pdf.

Hecker, A., « Le tramway a-t-il guidé la croissance urbaine au XIXe s. ? L'exemple de Nancy », Revue géographique de l'Est, vol. 57, nºs 3-4, 2017c, mis en ligne en octobre 2018, URL : http:// journals.openedition.org/rge/7243.

Husson, J.-P., « Voies d'eau et respiration urbaine. Réflexions appliquées à Metz et Nancy », Académie de Stanislas, 2019, URL : http://www.academie-stanislas.org/academiestanislas/ images/Colloques/Husson.pdf.

Husson, J.-P., « Legs et héritages des hydrosystèmes de défense et actuels projets de ville à Nancy », Le Pays Lorrain, $\mathrm{n}^{\circ}$ 1, 2018, p. 351-360.

Lechner, G., « Le Fleuve dans la ville. La valorisation des berges en milieu urbain », Paris, direction générale de l'Urbanisme, de l'Habitat et de la Construction, Centre de documentation de l'urbanisme, 2006, 118 p, URL : http://www.cdu.urbanisme.developpement-durable.gouv.fr/ IMG/pdf/fleuvedanslavilleaveccouv_cle24aafe.pdf.

Leguay, J.-P., L'Eau dans la ville au Moyen Âge, Rennes, Presses universitaires de Rennes, coll. « Histoire », 2002, publié en ligne en juillet 2015, URL : https://books.openedition.org/pur/23725.

Lévêque, C., Quelles rivières pour demain? Réflexions sur l'écologie et la restauration des cours d'eau, Versailles, Éditions Quæ, 2016, 288 p.

Lespez, L., Germaine, M.-A., « La rivière désaménagée ? Les paysages fluviaux et l'effacement des seuils et des barrages en Europe de l'Ouest et en Amérique du Nord-Est », BSGLg, vol. 67, n² 2, 2016, URL : https://popups.uliege.be:443/0770-7576/index.php?id=4465.

Mathis, D., Hecker, A., Husson, J.-P., Bensaadi, K., « Nancy et la Meurthe : la réconciliation patrimoniale d'un couple marqué par une défiance historique ", dans Carcaud, N., ArnaudFassetta, G., Évain, C. (dir.), Villes et Rivières de France, Paris, CNRS Éditions, 2019, p. 156-163.

Mathis, D., Mathis, A., « Transitions urbaines des anciennes villes de garnison en Allemagne : l'exemple de Landau in der Pfalz ", Revue géographique de l'Est, vol. 57, n 3-4, 2017, mis en ligne en avril 2019, URL : http://journals.openedition.org/rge/7526.

Mathis, D., « Les hydrosystèmes militaires défensifs de Basse-Alsace (XVII ${ }^{\mathrm{e}}-\mathrm{XVIII}{ }^{\mathrm{e}}$ siècles) », Revue de géographie historique, $\mathrm{n}^{\circ}$ 8, « Géographie historique et questions militaires » (1) », 2016, URL : 
http://rgh.univ-lorraine.fr/articles/view/70/

Les_hydrosystemes_militaires_defensifs_de_Basse_Alsace_XVIIe_XVIIIe_siecles.

Mathis, A., « Quand l'arbre rencontre la pierre » Le Haut-du-Lièvre et les carrières Solvay Enjeux de rénovation, réhabilitation, requalification ", mémoire de master, université de Lorraine, Nancy, 2012, 162 p.

Michel, A., « La ville-paysage du XXI ${ }^{\mathrm{e}}$ siècle : une symbiose de l'architecture et de la nature », Raison publique, $\mathrm{n}^{\circ}$ 17, 2012/2, p. 121-139, URL : https://www.cairn.info/revue-raisonpublique1-2012-2-page-121.htm.

Nicod, J., « La crue de la Meurthe et de la Moselle, 28 au 31 décembre 1947 », L'Information géographique, vol. 13, $\mathrm{n}^{\circ} 1,1949$, p. 12-16.

Pons, F., « Utilisation de données anciennes pour la connaissance des risques de submersions marines », colloque, SHF « Nouvelles approches sur les risques côtiers », Paris, 30 au 31 janvier 2008, URL : http://cete-aix.fr/dwld/NUNIEAU/2007-11-16-SHF-PONS.pdf.

Prêcheur, C., « Nancy, rapport de l'actuelle structure urbaine et de l'ancienne structure agraire », $B A G F, n^{\circ} 235-236,1953$, p. 106-114.

Prominski, M., Stokman, A., Zeller, S., Stimberg, D.,Voermanek, H., River. Space. Design. Planning strategies, methods and projects for urban rivers, Bâle, Birkhauser Verlag AG, 2012.

Rode, S., « Reconquérir les cours d'eau pour aménager la ville », Cybergeo. Revue européenne de géographie, document 806, mis en ligne en janvier 2017, URL : http://journals.openedition.org/ cybergeo/27933, DOI : 10.4000/cybergeo.27933.

Salles, S., « Contraintes environnementales et opportunités paysagères : Nancy "Rives de Meurthe" ", Espaces et Sociétés, n 146, 2011/3, p. 53-59, URL : https://www.cairn.info/revueespaces-et-societes-2011-3-page-53.htm, DOI : 10.3917/esp.146.0053.

Scherrer, F., "L'eau urbaine ou le pouvoir de renaturer », Cybergeo. Revue européenne de géographie, mis en ligne en décembre 2004, URL : http://journals.openedition.org/cybergeo/1496, DOI : 10.4000/cybergeo.1496

Sgard, A., « Entre rétrospective et prospective. Comment reconstruire le récit du territoire? ", EspacesTemps.net, septembre 2008, URL : https://www.espacestemps.net/articles/entreretrospective-et-prospective/.

Valette, P., Carozza, J.-M., « Mise en œuvre d'une démarche géohistorique pour la connaissance de l'évolution des paysages fluviaux : l'exemple de la moyenne vallée de la Garonne », Géocarrefour, vol. 85/1, 2010, p. 17-27, mis en ligne en mai 2010, URL : http:// journals.openedition.org/geocarrefour/7679; DOI : 10.4000/geocarrefour.7679.

\section{NOTES}

1. Par hydrosystème défensif, Denis Mathis (2016) définit pour le XVIII ${ }^{\mathrm{e}}$ siècle « des fortifications de campagnes semi-permanentes organisées le long des cours d'eau. Elles associent des retranchements de terres couvertes de palissades afin d'assurer la protection des hommes et l'aménagement de petits bassins hydrographiques destinés à organiser un obstacle "naturel" non guéable et inondable ".

2. Arrivée du canal de la Marne au Rhin qui précède de peu celle du chemin de fer (1850-1852).

3. Cette courte période correspond à la fois à la succession d'une crue trentennale (1982) et de deux crues cinquantennales (avril et mai 1983). 
4. À l'époque des Trente Glorieuses, dans un contexte d'optimisme économique en faveur de l'essor industriel, avait été envisagé le passage à gabarit européen de la Meurthe, depuis sa confluence à Frouard jusqu'aux salines de Varangéville. Ceci ne se fit pas mais les préétudes établies servirent de base pour engager les travaux qui se sont imposés afin de se prémunir des effets des trois crues de décembre 1982, d'avril et de mai 1983.

5. Au XI ${ }^{\mathrm{e}}$ siècle, le château de Gérard d'Alsace est à l'origine de la ville.

6. La porte monumentale fut déplacée lors de la construction de la caserne Thiry pour intégrer celle-ci au périmètre urbain.

7. La situation de "ville frontière ", au sein d'un espace régional redéfini, fait de Nancy et de sa région le réceptacle du repli des activités économiques des «provinces perdues » qui veulent conserver leurs liens nationaux et commerciaux avec la France.

8. La paysagiste Claire Alliod a équipé le projet ARTEM de mares et jardins aquatiques.

9. Le site de l'ancienne cartonnerie de la Rochette est en cours de retraitement. Il tient compte du classement aux monuments historiques du bâtiment de la chaufferie (façades, toitures, structures et portique de la chaufferie centrale thermique (cad. AC 512) : inscription par arrêté du 17 février 2012, URL: http://www2.culture.gouv.fr/public/mistral/merimee_fr? ACTION=CHERCHER\&FIELD_1=REF\&VALUE_1=PA54000075.

10. Son régime pluvionival est identique à celui de la Meurthe.

11. La Seille a été intégrée au périmètre de défense messin puis a été transformée en hydrosystème de défense par inondation.

\section{RÉSUMÉS}

Cette étude observe et analyse les relations géohistoriques au sein des couples villes-fleuves et de l'hydromorphologie urbaine à l'échelle du Sillon lorrain. Ces relations fondées souvent sur la défiance dans une démarche d'aménagement évoluent aujourd'hui vers une approche plus équilibrée que par le passé, construite sur la logique de ménagement des cours d'eau et d'une relation plus apaisée. Ce nouvel itinéraire implique une transition paysagère en construction des couples villes-fleuves. L'interface rivulaire est valorisée, facilitant les opérations de réhabilitation ou de régénération urbaine, transposées en tenant compte des héritages et des spécificités des villes de Nancy, de Metz et de Thionville. Cette nouvelle approche s'appuie sur un « récit » et une «mémoire » qui servent la réconciliation et la construction ou la consolidation des fronts d'eau urbains.

This study observes and analyses geohistorical relationships within cities and their rivers and urban hydromorphology at the scale of the Sillon Lorrain urban cluster. These relationships, which were often conditioned by mistrust within an urban development context, are now evolving towards a more balanced approach to planning based on the logic of river management and are thus towards a more harmonious relationship. This new approach involves a transition in landscape planning involving the pairing of urban centres and their rivers. Riversides are enhanced, facilitating transpositions of urban rehabilitation or regeneration operations which account for the heritages and unique qualities of the cities of Nancy, Metz and Thionville. This new approach is based on a "narrative" and on "memory" which are put at the service of reconciliation and the construction or consolidation of urban waterfronts. 
INDEX

Keywords : Lorraine trench, vulnerability, urban hydromorphology, waterfront, landscape transition

Mots-clés : Sillon lorrain, vulnérabilité, hydromorphologie urbaine, front d'eau, transition paysagère

\section{AUTEURS}

\section{DENIS MATHIS}

Denis Mathis est maître de conférences, université de Lorraine, LOTERR. Ses domaines de recherche : géohistoire, dynamiques paysagères, géographie rurale, territoires d'eau, territoires militarisés, transitions et reconversions.

denis.mathis[at]univ-lorraine[dot]fr

\section{ANNE HECKER}

Anne Hecker est maître de conférences en géographie, université de Lorraine, LOTERR. Ses domaines de recherche : mobilités, géohistoire, évolution des paysages, transport et forme urbaine, SIG historique.

anne.hecker[at]univ-lorraine[dot]fr

https://loterr.univ-lorraine.fr/content/hecker-anne

\section{JEAN-PIERRE HUSSON}

Jean-Pierre Husson est professeur émérite des universités, université de Lorraine, LOTERR. Ses domaines de recherche : aménagement rural, qualité, aménité, artialisation. husson18[at]univ-lorraine[dot]fr

\section{KAMILA BENSAADI}

Kamila Bensaadi est doctorante en géographie et chargée de la valorisation de la recherche, université de Lorraine, LOTERR. Ses domaines de recherche : karst, risque, vulnérabilité, aléa, effondrements, inondation, aménagement du territoire, Barrois.

Kamila.bensaadi[at]univ-lorraine[dot]fr

https://geonancy.wordpress.com/enseignants-chercheurs/kamila-bensaadi/ 